\title{
Ibuprofen does not Impair Skeletal Muscle Regeneration Upon Cardiotoxin-Induced Injury
}

\author{
Sebastiaan DALLE ${ }^{1}$, Chiel POFFÉ ${ }^{1}$, Charlotte HIROUX ${ }^{1}$, Frank SUHR ${ }^{1}$, Louise \\ DELDICQUE $^{2}$, Katrien KOPPO ${ }^{1}$
}

${ }^{1}$ Exercise Physiology Research Group, Department of Movement Sciences, Catholic University of Leuven, Leuven, Belgium, ${ }^{2}$ Institute of Neuroscience, Université Catholique de Louvain, Louvainla-Neuve, Belgium

Received April 9, 2020

Accepted July 8, 2020

Epub Ahead of Print September 9, 2020

\section{Summary}

Muscle regeneration is regulated through interaction between muscle and immune cells. Studies showed that treatment with supra-physiological doses of Non-Steroidal Anti-Inflammatory Drug (NSAID) abolished inflammatory signaling and impaired muscle recovery. The present study examines the effects of pharmacologically-relevant NSAID treatment on muscle regeneration. C57BL/6 mice were injected in the tibialis anterior (TA) with either PBS or cardiotoxin (CTX). CTX-injected mice received ibuprofen (CTX-IBU) or were untreated (CTX-PLAC). After 2 days, $I /-1 \beta$ and $I /-6$ expression was upregulated in the TA of CTX-IBU and CTX-PL vs. PBS. However, Cox-2 expression and macrophage infiltration were higher in CTX-PL vs. PBS, but not in CTX-IBU. At the same time, anabolic markers were higher in CTX-IBU vs. PBS, but not in CTX-PL. Nevertheless, ibuprofen did not affect muscle mass or muscle fiber regeneration. In conclusion, mild ibuprofen doses did not worsen muscle regeneration. There were even signs of a transient improvement in anabolic signaling and attenuation of inflammatory signaling.

\section{Key words}

NSAID • Inflammation • mTORC1 signaling • Cyclooxygenase • Muscle recovery

\section{Corresponding author}

K. Koppo, Exercise Physiology Research Group, Department of Movement Sciences, Faculty of Movement and Rehabilitation Sciences, Catholic University of Leuven, Tervuursevest 101, B-3001 Leuven, Belgium. E-mail: katrien.koppo@kuleuven.be

\section{Introduction}

Skeletal muscle injuries frequently occur, e.g. due to work or traffic accidents or sport traumas. If the muscle tissue is incompletely or improperly regenerated, muscle capacity can be permanently impaired, which might limit daily-living activities. The muscle recovery process is closely regulated by inflammatory signaling, e.g. cytokines produced by neutrophils and macrophages, which modulate other immune cells, myocytes and their interaction. The cytokines and mitogens that are released in the injured skeletal muscle tissue promote the synthesis of prostaglandins (PGs) in inflammatory cells. Besides their modulatory role in inflammation, PGs are involved in muscle metabolism, and more specifically in myoblast proliferation, differentiation and fusion (Leng and Jiang 2019, Mo et al. 2015), and in the regulation of muscle protein synthesis (Weinheimer et al. 2007) and degradation (Markworth and Cameron-Smith 2011, Rodemann et al. 1982, Vandenburgh et al. 1990). Therefore, PGs have gained much interest as mediators of the skeletal muscle regeneration process.

There are many therapies available to modulate muscle regeneration, including RICE (rest, ice, compression and elevation), physical therapy and medication. Especially, non-steroidal anti-inflammatory drugs (NSAIDs) are very commonly used for muscle regeneration purposes (Elnachef et al. 2008). Through COX-inhibition, NSAIDs attenuate the PG production, and thereby dampen inflammatory signaling (Green 2001). Although very popular, NSAIDs play a dual role 
in muscle regeneration. Shortly upon injury, NSAIDs seem to induce a more complete functional recovery. Mishra et al. (1995) observed faster muscle strength regeneration in rabbits when an exercise-induced muscle injury was treated with flurbiprofen (Mishra et al. 1995). However, this effect was only temporary ( $\sim 7$ days). On day 28, NSAID treatment resulted in a deficit in muscle strength compared to the untreated group (Mishra et al. 1995). This indicates that the effect of NSAID treatment on skeletal muscle tissue is not as straightforward as often suggested. Indeed, NSAIDs might improve the performance upon injury, through inhibition of the initial inflammatory reaction and concomitant pain.

Yet, the role of NSAID-induced inhibition of the inflammatory response in muscle regeneration remains controversial (Järvinen et al. 2013, Mishra et al. 1995, Prisk and Huard 2003). Two rodent studies raised caution about the use of COX inhibitors in the context of muscle recovery, as they showed that COX inhibition impaired the skeletal muscle regeneration process, mainly via a dysregulated inflammatory signaling (Bondesen et al. 2004, Novak et al. 2009). However, in both studies, COX signaling was inhibited to a supra-physiological extent, either pharmacologically or via COX knockout. However, a minimal inflammatory response by immune cells (i.e. phagocytosis of cellular debris and the release of chemoattractants and growth factors) facilitates myogenesis during regeneration (Chazaud et al. 2003, Tidball 2005). Therefore, we hypothesize that a mild, pharmacologically-relevant COX inhibition, that only slightly attenuates the inflammatory response, does not impair muscle regeneration.

The present study examines whether mild NSAID treatment affects the skeletal muscle regeneration process upon cardiotoxin-induced muscle injury, through changes in muscle histology, muscle anabolic signaling
(mTORC1) and muscle inflammatory signaling in C57BL/6 mice.

\section{Methods}

\section{Animal use}

The experiment was approved by the KU Leuven Animal Ethics Committee (P168/2016), and all methods were performed in accordance with the relevant guidelines and regulations. Seventy-two young (10 weeks) male C57BL/6 mice were purchased from Janvier Labs (Le Genest-Saint-Isle, France). Mice were supplemented with either ibuprofen $\left(20 \mathrm{mg} \cdot \mathrm{kg}^{-1} \cdot \mathrm{d}^{-1}\right)$, dissolved in liquid diet or liquid diet (F1259SP, Bio-Serve; Flemington, USA) as such. To allow appropriate ibuprofen treatment, the liquid diet of individually housed mice was daily weighed and ibuprofen doses in the liquid diet were adapted to achieve $20 \mathrm{mg} \cdot \mathrm{kg}^{-1} \cdot \mathrm{d}^{-1}$. Three days following the start of the supplementation, the muscle Tibialis Anterior (TA) of both hindlimbs of the ibuprofen-treated group $(n=24$; CTX-IBU) and of half of the placebo group $(n=24$; CTX-PL) was injected with CTX $(10 \mu \mathrm{M}$ in $150 \mu \mathrm{l}$ phosphate buffered saline (PBS); L8102, Latoxan, Porteslès-Valence, France), while the other half of the placebo group was injected with PBS (control group) $(n=24)$. Prior to the injection, mice were anaesthetized by intraperitoneal injection of $10 \mu \mathrm{l} \cdot \mathrm{g}^{-1} \mathrm{BM}$ of saline solution containing xylazine $\left(100 \mathrm{mg} \cdot \mathrm{ml}^{-1}\right.$, Rompun $5 \%$ ) and ketamine $\left(100 \mathrm{mg} \cdot \mathrm{ml}^{-1}\right.$, Nimatek $\left.10 \%\right)$. In each condition, 8 mice were sacrificed respectively 2, 5 and 12 days following CTX- or PBS-injection. TA muscles were surgically removed, weighed and snap frozen in liquid nitrogen and stored at $-80^{\circ} \mathrm{C}$ for biochemical and histological analyses. A schematic overview of the study design is presented in Fig. 1.

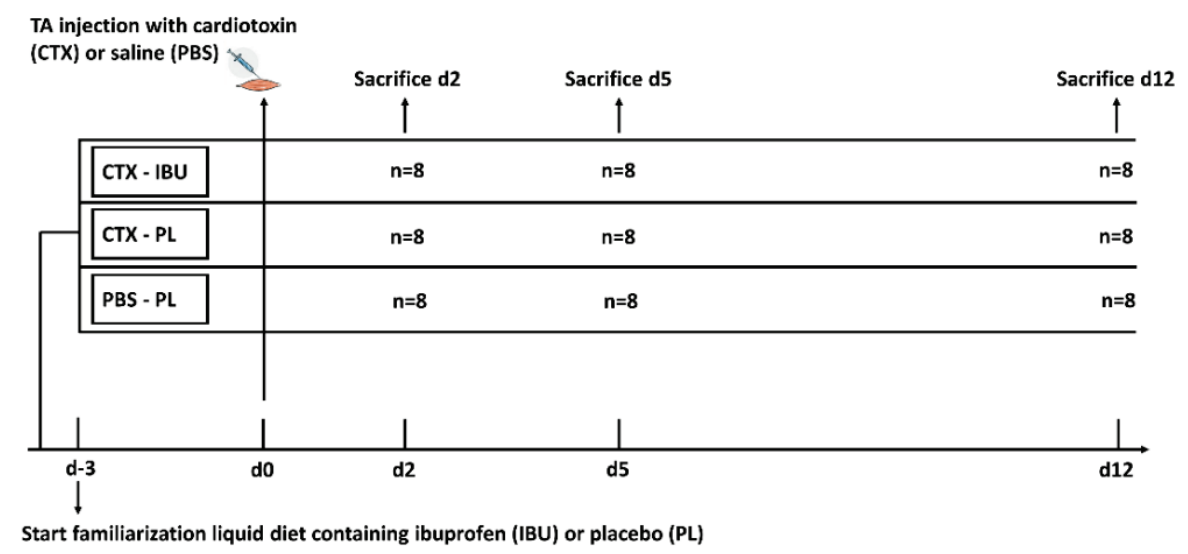

Fig. 1. Schematic overview of the study protocol. 


\section{Histological analyses}

Muscle tissues were embedded in tissue freezing medium (Leica Biosystems, Wetzlar, Germany) and frozen in liquid nitrogen-cooled isopentane. Serial cryosections ( $7 \mu \mathrm{m}$ thick) were cut with a cryostat (Leica Biosystems CM1850, Wetzlar, Germany) at $-20{ }^{\circ} \mathrm{C}$. Prior to histological analyses, cryosections were thawed at room temperature (RT), washed with PBS and fixed with $4 \%$ paraformaldehyde. To permit qualitative analysis of muscle fiber morphological alternations, cryosections were stained with haematoxylin and eosin (H\&E) (Sigma Aldrich, St Louis, USA). In addition, to assess muscle regeneration, the characteristics of myofibers with a centralized nucleus were measured in the entire muscle section of mice in CTX-PL and CTX-IBU at $\mathrm{d} 5$, as this time point corresponds with maximal regeneration (Chen et al. 2005, Garry et al. 2016). As expected, no fibers with a centralized nucleus were observed in PBS. For the muscle fiber type composition and F4/80-positive $\left(\mathrm{F} 4 / 80^{+}\right)$macrophage staining, cryosections were blocked for $2 \mathrm{~h}$ in PBS containing $1 \%$ bovine serum albumin (BSA). Following permeabilization in PBS (1\% BSA + $0.2 \%$ Triton $\mathrm{X}-100)$ for $15 \mathrm{~min}$, cryosections were incubated overnight at $4{ }^{\circ} \mathrm{C}$ in a humid room with primary antibodies; for fiber typing (Developmental Studies Hybridoma Bank, Iowa, USA): BA-F8 (1:400, myosin heavy chain (MyHC) I), SC-71 (1:100, MyHC IIa), BF-F3 (1:300, MyHC IIb) and L9393 (1:500, Laminin, Sigma Aldrich, St Louis, USA); for macrophage staining (Cell Signaling Technology, Leiden, The Netherlands): F4/80 (1:250; D2S9R) dissolved in PBS. After washing in PBS, cryosections were incubated for $1 \mathrm{~h}$ at RT with the conjugated secondary antibodies; for fiber typing (Life Technologies, California, USA): goat anti-mouse Alexa-488 IgG2 (1:300, MyHC I), goat anti-mouse Alexa-350 IgG1 (1:300, MyHc IIa), goat antimouse Alexa-594 IgM (1:300, MyHC IIb), donkey antirabbit Alexa-488 $\operatorname{IgG}$ (1:600, laminin); for macrophage staining: donkey anti-rabbit Alexa-488 IgG (1:300, $\mathrm{F} 4 / 80$ ).

Slides were visualized by fluorescence microscopy (Nikon E1000, Nikon, Boerhavedorp, Germany). The epifluorescence signal was recorded with FITC (MyHC I, cell membranes and $\mathrm{F} 4 / 80^{+}$macrophages), DAPI (MyHC IIa) and Texas Red (MyHC IIb) excitation filters. Muscle fibers of the entire muscle section were classified as type I, type IIa, type IIb or immature + type IIx (unstained fibers). The $\mathrm{F} 4 / 80^{+}$macrophages infiltration was quantified by the mean color density of the whole muscle cryosection. Muscle cryosections were analyzed with ImageJ software (version 1.41, National Institutes of Health, USA) by investigators who were blinded to the experimental conditions.

\section{Protein extraction}

Muscle samples were homogenized with a mortar, dissolved in ice-cold lysis buffer [1:10, w/v; $50 \mathrm{mM}$ Tris- $\mathrm{HCl}, \quad$ pH 7.0; $270 \mathrm{mM}$ sucrose; $5 \mathrm{mM}$ EGTA; $1 \mathrm{mM}$ EDTA; $1 \mathrm{mM}$ sodium orthovanadate; $50 \mathrm{mM}$ glycerophosphate; $5 \mathrm{mM}$ sodium pyrophosphate; $50 \mathrm{mM}$ sodium fluoride; $1 \mathrm{mM}$ dithiothreitol; $0.1 \%$ Triton X-100; and a complete protease inhibitor tablet (Roche Applied Science, Vilvoorde, Belgium)] and centrifuged for $25 \mathrm{~min}$ at $10000 \times \mathrm{g}$ at $4{ }^{\circ} \mathrm{C}$. The supernatant was aliquoted and stored at $-80{ }^{\circ} \mathrm{C}$. The protein concentration was assessed with the $\mathrm{DC}$ protein assay kit applying a BSA protein standard (Bio-Rad Laboratories, Nazareth, Belgium). Lysis buffer was added to equalize protein concentrations. Laemmli ( $20 \%$ of the total volume) was added to obtain muscle lysates.

\section{Western blot analyses}

The lysate protein content $(30-50 \mu \mathrm{g})$ was separated using an SDS-PAGE gel (8-12\% sodium acrylamide) and were transferred to polyvinylidene difluoride membranes, which were next blocked in Trisbuffered saline Tween-20 (TBS-T) with $5 \%$ BSA for $1 \mathrm{~h}$ and incubated with the primary antibody, dissolved 1:200-1:10000 in $5 \%$ BSA in TBS-T, at $4{ }^{\circ} \mathrm{C}$ overnight: phospho-Akt (Ser473; CST5171), phospho-mTOR (Ser2448; CST2971S), phospho-S6 Kinase (Thr389; CST9206S), GAPDH (CST2118S) (Cell Signaling Technology, Leiden, The Netherlands). Secondary antimouse (1:7000) and anti-rabbit (1:5000) antibodies conjugated to horseradish peroxidase were applied to detect target proteins. Target protein bands were quantified with the GeneSnap software and tools (Syngene, Cambridge, UK). Since CTX-injection increased the total protein form of mTOR and S6K1 compared to PBS, proteins were presented relative to GAPDH.

\section{$R N A$ extraction and real-time quantitative $P C R$}

Total RNA was extracted from $\sim 20 \mathrm{mg}$ of muscle tissue, homogenized in $1 \mathrm{ml}$ Trizol reagent (Thermo Fisher Scientific, Waltham, USA). The RNA purity and concentration were assessed by 
Nanodrop (Thermo Fisher Scientific) spectrophotometry. Reverse transcription was performed from $1 \mu \mathrm{g}$ RNA using the iScriptcDNA Synthesis Kit (Bio-Rad Laboratories, Hercules, USA), following the manufacturer's instructions. The PCR was run using the following conditions: $2 \mathrm{~min}$ at $95^{\circ} \mathrm{C}$, followed by 50 cycles of $15 \mathrm{~s}$ at $95^{\circ} \mathrm{C}, 30 \mathrm{~s}$ at $60^{\circ} \mathrm{C}$ and $40 \mathrm{~s}$ at $72{ }^{\circ} \mathrm{C}$. Each reaction was processed in a $25 \mu 1$ volume containing $12.5 \mu 1$ Promega PCR Master Mix, $1 \mu 1$ of each primer $(0.1 \mu \mathrm{M}), 8.5 \mu \mathrm{l}$ RNAse free $\mathrm{H}_{2} \mathrm{O}$ and $2 \mu \mathrm{l}$ cDNA (25 ng). Mouse-specific primers for Cyclooxygenase-2 (Cox-2), Interleukin (Il)- 6 and $I l-1 \beta$ were designed (Table 1). To compensate for variations in input RNA amounts and efficiency of reverse transcription, ribosomal protein L41 (rp141) mRNA was quantified, and results were normalized to those values using the $\Delta \Delta \mathrm{C}_{\mathrm{T}}$ method. The results are expressed in arbitrary units.

Table 1. COX-2: cyclooxygenase-2, IL: interleukin, Rpl41: ribosomal protein L41.

\begin{tabular}{lcccc}
\hline Gene & Forward & Reverse & Template & $\begin{array}{c}\text { Product } \\
\text { size }\end{array}$ \\
\hline Cox-2 & ATGGGTGTGAAGGGAAATAAGGA & CCTTGGGGGTCAGGGATGAA & NM_011198.4 & 92 \\
$I l-6$ & GACAAAGCCAGAGTCCTTCAGA & GTGACTCCAGCTTATCTCTTGGT & NM_001314054.1 & 75 \\
$I l-1 \beta$ & ATGCCACCTTTTGACAGTGATG & GCAGCCCTTCATCTTTTGGG & NM_008361.4 & 72 \\
Rpl41 & ATGAGAGCGAAGTGGCGGAAG & CAGGGCAGAGGGACTGTTTTG & NM_018860.4 & 260 \\
\hline
\end{tabular}

\section{Statistical analyses}

All values are presented as average \pm SEM. A two-way ANOVA across conditions and time was performed. Post hoc analyses were Tukey-corrected and statistical significance was accepted for $\mathrm{p}<0.05$. All statistical analyses were completed with SPSS (Version 22.0.0.0; IBM Corp, NY, USA).

\section{Results}

\section{Muscle injury and regeneration}

CTX-injection effectively induced muscle injury and regeneration, as the destruction, repair and remodeling phase were clearly distinguishable at 2 (d2), 5 (d5) and 12 (d12) days following the injection, both in untreated (CTX-PL) and ibuprofen-treated (CTX-IBU) group (Fig. 2). The early response upon CTX-induced skeletal muscle injury (d2) was characterized by inflammatory cell infiltration and interruption of the myofibers' integrity. At $\mathrm{d} 5$, inflammatory cells remained present and new, small regenerating myofibers with centralized nuclei appeared. The relative contribution of fibers with a centralized nucleus ( $\%$ to the total fiber number) did not differ between CTX-PL $(42.6 \pm 6.4 \%)$ and CTX-IBU $(36.5 \pm 3.2 \% ; \mathrm{p}=0.44)$, neither did the mean cross sectional area $\left(667 \pm 34 \mu \mathrm{m}^{2}\right.$ in CTX-PL and $663 \pm 45 \mu \mathrm{m}^{2}$ in CTXIBU; $\mathrm{p}=0.94)$ and perimeter $(99 \pm 3 \mu \mathrm{m}$ in CTX-PL and $98 \pm 3 \mu \mathrm{m}$ in CTX-IBU; $\mathrm{p}=0.83$ ) of fibers with a centralized nucleus. Twelve days following CTX-injection, myofiber integrity was partially restored and regenerating myofibers with centralized nuclei were enlarged compared to $\mathrm{d} 5$.

\section{Muscle mass}

The mass of the non-injected hind limb muscles (gastrocnemius, soleus, quadriceps) was unaffected by condition or time. TA muscle mass was not different between conditions at $\mathrm{d} 2$ and $\mathrm{d} 12$, while at $\mathrm{d} 5$, TA mass was significantly lower in CTX-injected conditions compared to the PBS-injected controls (CTX-PL: $40.1 \pm 0.9 \mathrm{mg}$; CTX-IBU: $40.1 \pm 2.4 \mathrm{mg}$; PBS: $50.6 \pm 1.0 \mathrm{mg}$; $\mathrm{p}<0.001)$. There was no difference in TA muscle mass between CTX-PL and CTX-IBU at the different time points.

Muscle fiber composition (Table 2; Fig. 3)

CTX-injection induced a shift in muscle fiber type composition. Two days following CTX-injection, there was a $\sim 10 \%$ loss of type IIa muscle fibers compared to PBS, irrespective of treatment (CTX-PL: $p=0.006$; CTXIBU: $p=0.032$; Fig. 3A), while unstained muscle fibers (immature + type IIx) increased upon CTX-injection ( $+30 \%$ in CTX-PL, $p<0.001 ;+20 \%$ in CTX-IBU, $p=0.02$; Fig. 3C). Type IIb muscle fibers decreased in CTX-PL $(-17.1 \%$ vs. PBS; $p=0.025)$, but not in CTX-IBU $(p=0.398$; Fig. 3B). Five days upon CTX-injection, type IIa muscle fibers remained low in CTX-PL $(-12.5 \% ; p=0.007)$ and CTX-IBU (-10.0\%; $=0.041$; Fig. $3 \mathrm{~A})$ vs. PBS, while unstained fibers (Fig. 3C) and type IIb muscle fibers 
(Fig. 3B) did not differ between conditions. At d12, type IIa muscle fibers tended to be lower in CTX-PL compared to PBS $(-17 \% ; \mathrm{p}=0.10)$, whereas this was not the case for CTX-IBU (-10\%; Fig. 3A). In line with d5, unstained and type IIb fibers were similar between conditions (Fig. 3B, C). At none of the time points and for none of the fiber types, there were significant differences between the CTX-PL and CTX-IBU condition ( $\mathrm{p}>0.05$ ).

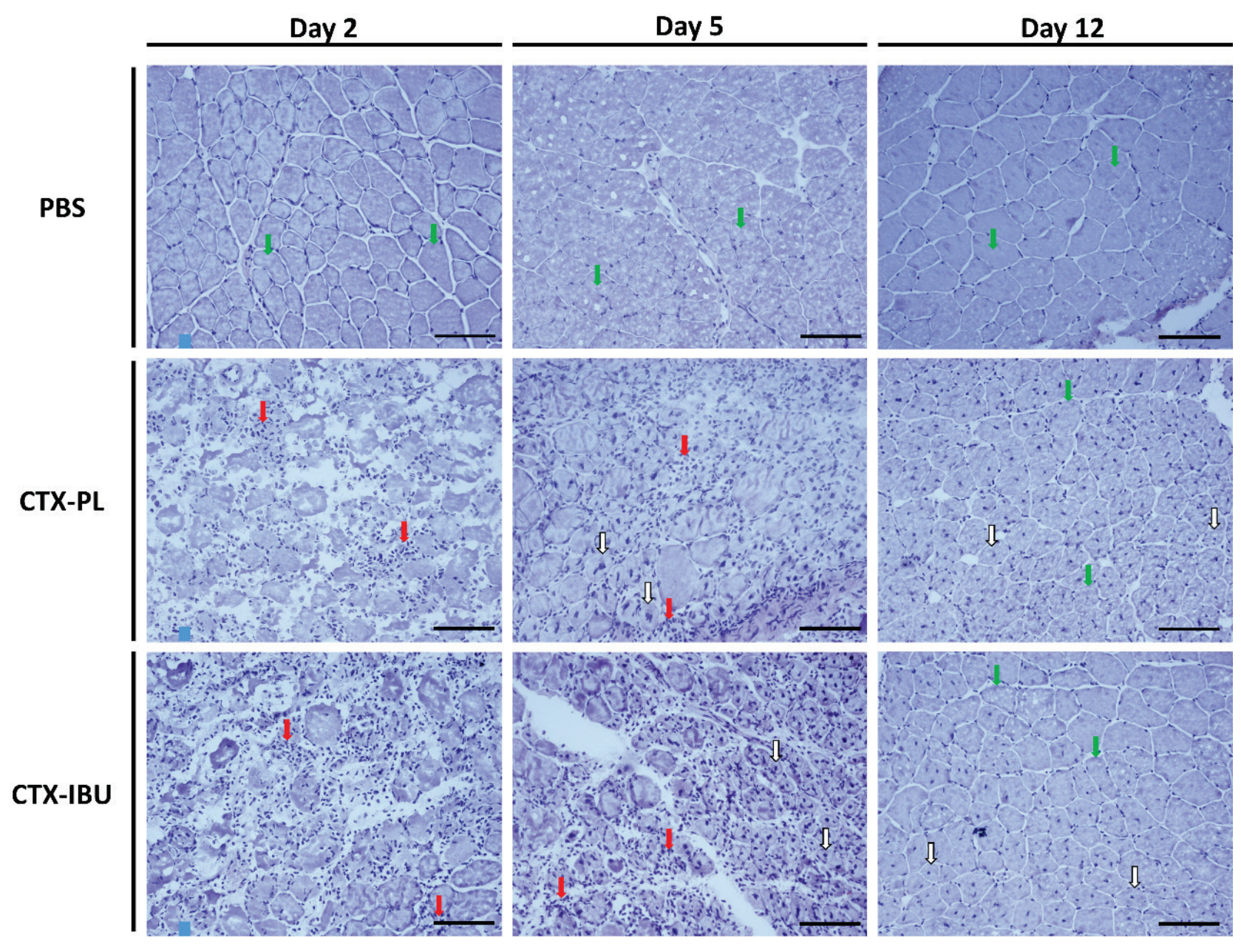

Fig. 2. Regeneration phases of TA muscle fibers. Histological sections stained with $H \& E$ revealed muscle infiltration of immune cells (red arrows) on day 2 (myolysis) and day 5 (early regeneration) following CTX injection. At d5, damaged myofibers were replaced by small newly formed myofibers with a centralized nucleus (white arrows). Twelve days upon CTX injury, myofibers with a centralized nucleus (white arrows) were enlarged and 'healthy' myofibers with a nucleus in the periphery appeared (green arrows). PBS: healthy controls (PBS-injected), CTX-PL: CTX-injected and untreated, CTX-IBU: CTX-injected and ibuprofen-treated. Scale bar=100 $\mu \mathrm{m}$.

Table 2. Data are average \pm SEM for relative fiber number (\%) in the muscle Tibialis Anterior. PBS: healthy controls (PBS-injected), CTX-PL: CTX-injected and untreated, CTX-IBU: CTX-injected and ibuprofen-treated. ${ }^{*} p<0.05 ;{ }^{+} p=0.05-0.1$ compared to PBS within same day following sacrifice.

\begin{tabular}{lccc|ccc|ccc}
\hline & \multicolumn{3}{c|}{ Day 2 } & \multicolumn{3}{c|}{ Day 5 } & \multicolumn{3}{c}{ Day 12 } \\
\cline { 2 - 10 } $\begin{array}{l}\text { Relative } \\
\text { contribution (\%) }\end{array}$ & PBS & CTX-PL & $\begin{array}{c}\text { CTX- } \\
\text { IBU }\end{array}$ & PBS & $\begin{array}{c}\text { CTX- } \\
\text { PL }\end{array}$ & $\begin{array}{c}\text { CTX- } \\
\text { IBU }\end{array}$ & PBS & CTX-PL & CTX-IBU \\
\hline Type I & $0.04 \pm 0.03$ & $0.04 \pm 0.03$ & $0.04 \pm 0.04$ & $0.02 \pm 0.02$ & $0.10 \pm 0.08$ & $0.16 \pm 0.11$ & $0.06 \pm 0.05$ & $0.06 \pm 0.04$ & $0.05 \pm 0.04$ \\
Type IIa & $17.4 \pm 3.9$ & $4.8 \pm 1.3^{*}$ & $7.1 \pm 1.7^{*}$ & $17.2 \pm 2.7$ & $4.7 \pm 2.1^{*}$ & $7.2 \pm 1.7^{*}$ & $19.4 \pm 4.2$ & $10.6 \pm 2.4^{\dagger}$ & $15.0 \pm 3.6$ \\
Type IIb & $54.8 \pm 5.6$ & $37.7 \pm 6.8^{*}$ & $45.2 \pm 6.3$ & $54.2 \pm 1.8$ & $56.2 \pm 2.6$ & $50.3 \pm 3.2$ & $56.1 \pm 4.0$ & $57.9 \pm 3.9$ & $56.5 \pm 2.9$ \\
Unstained fibers & $27.8 \pm 5.1$ & $57.4 \pm 7.6^{*}$ & $47.6 \pm 6.1^{*}$ & $28.6 \pm 2.7$ & $39.0 \pm 4.1$ & $42.4 \pm 3.1$ & $24.5 \pm 5.2$ & $31.4 \pm 5.3$ & $28.4 \pm 4.1$ \\
\hline
\end{tabular}


F4/80 $0^{+}$macrophage infiltration (Fig. 4)

Macrophage infiltration was increased at $\mathrm{d} 2$ both in CTX-PL and in CTX-IBU. However, the increase compared to PBS only reached significance in CTX-PL $(\mathrm{p}=0.029)$, while there was no significant difference
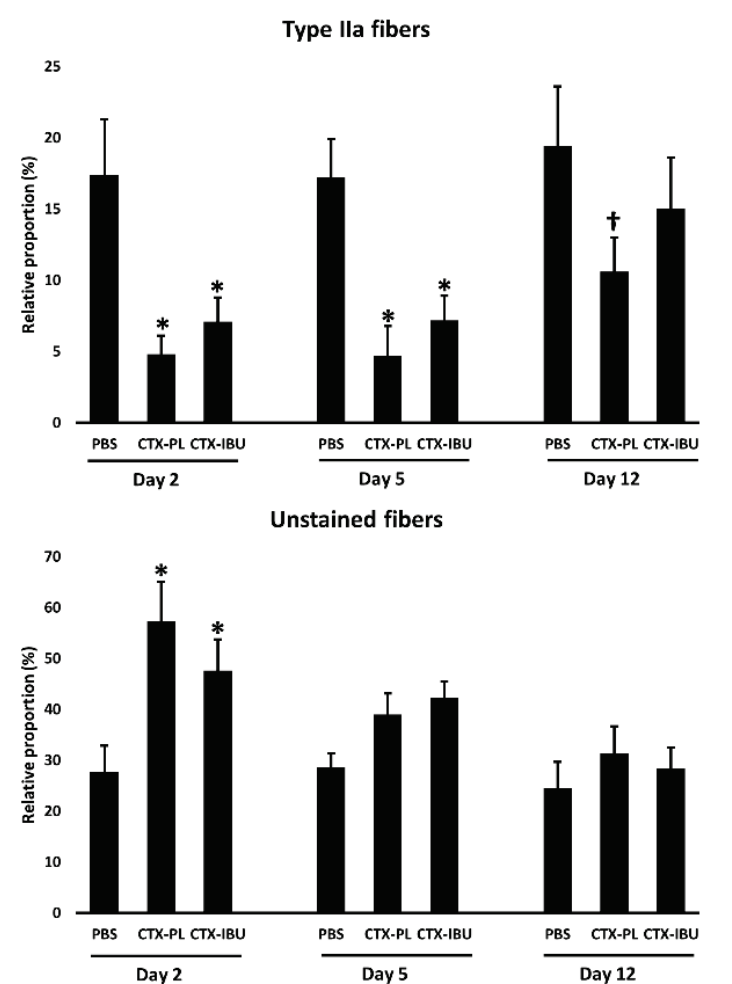

between CTX-IBU and PBS $(p=0.186)$. At $d 5$, CTX-injection increased macrophage infiltration, irrespective of treatment. At d12, macrophage infiltration in the CTX-injected conditions was comparable to PBS.

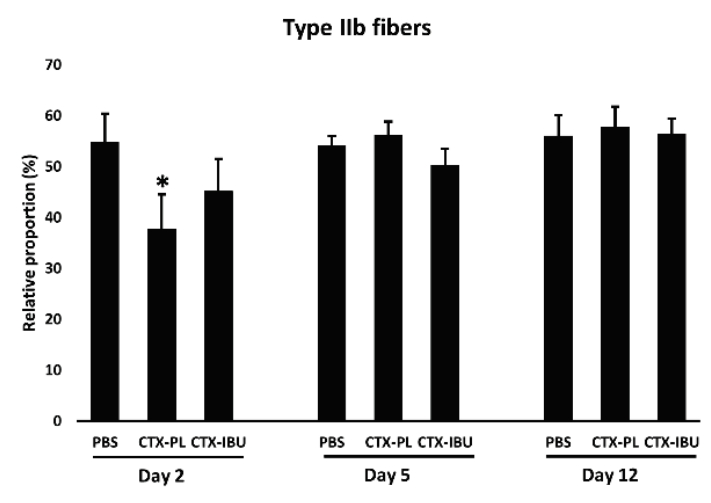

Fig. 3. Data are presented as average \pm SEM for the relative contribution (as \% of total fibers) of type IIa, type IIb and unstained (type IIx + immature) fibers of the muscle cross-section. PBS: healthy controls (PBS-injected), CTX-PL: CTX-injected and untreated, CTX-IBU: CTX-injected and ibuprofen-treated. ${ }^{*} p<0.05 ;{ }^{\dagger} p=0.05-0.1$.

\section{$\mathrm{F} 4 / 80^{+}$Macrophages}

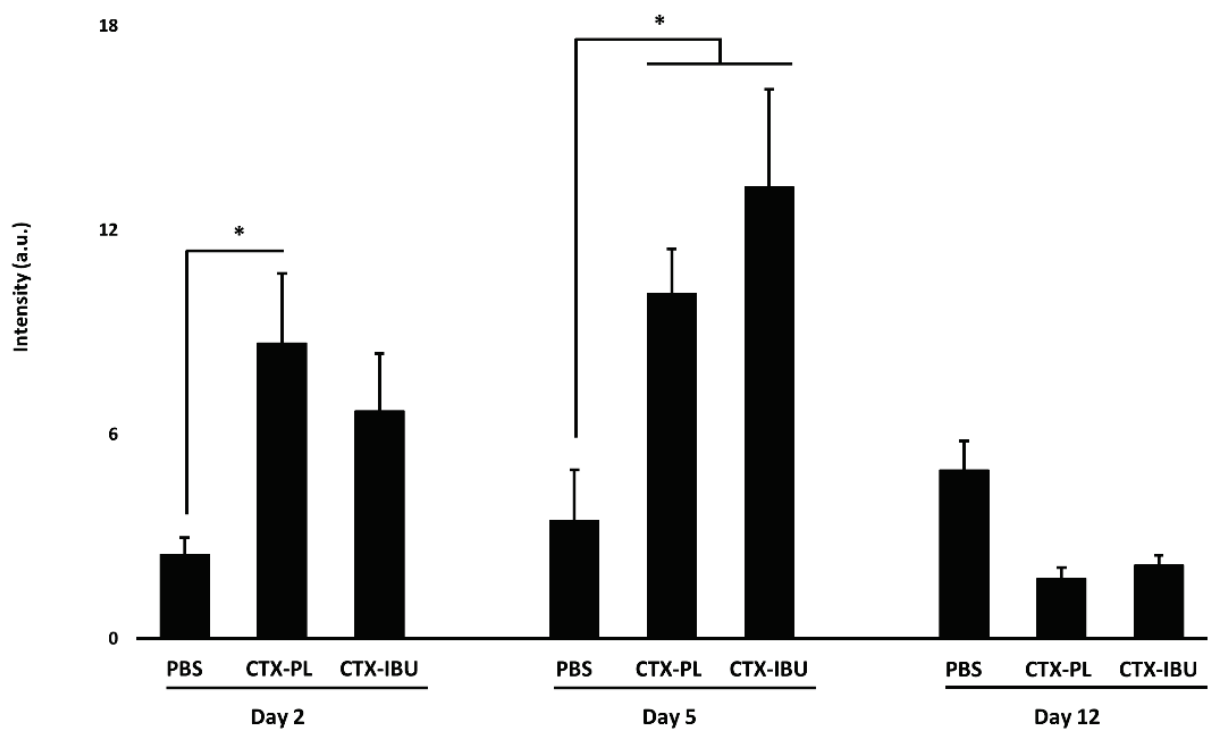

Fig. 4. Data are presented as average $\pm \mathrm{SEM}$ for $\mathrm{F} 4 / 80^{+}$macrophage infiltration. PBS: healthy controls (PBS-injected), CTX-PL: CTX-injected and untreated, CTX-IBU: CTX-injected and ibuprofen-treated. $* p<0.05$. 


\section{Muscle inflammation (Fig. 5)}

Upon CTX-injection, inflammatory signaling was increased compared to the PBS-injection. Cox-2 (Fig. 5A) mRNA expression at d2 was more elevated in CTX-PL (19.5 \pm 12.2 a.u.) vs. PBS (1.0 \pm 0.1 a.u.; $\mathrm{p}=0.017$ ), while this was not the case for CTX-IBU (12.0 \pm 3.0 a.u.; $p=0.251$ ). However, there was no significant difference between CTX-PL and CTX-IBU $(\mathrm{p}=0.68)$. At $\mathrm{d} 5$ and $\mathrm{d} 12, \operatorname{Cox}-2$ mRNA expression did not differ between the three conditions. Both Il-6 (Fig. 5B) and $I l-1 \beta$ (Fig. 5C) mRNA expression were elevated 2 days following CTX-injection, irrespective of treatment. Similarly to Cox-2, their expression was not different from PBS at $\mathrm{d} 5$ and $\mathrm{d} 12$.
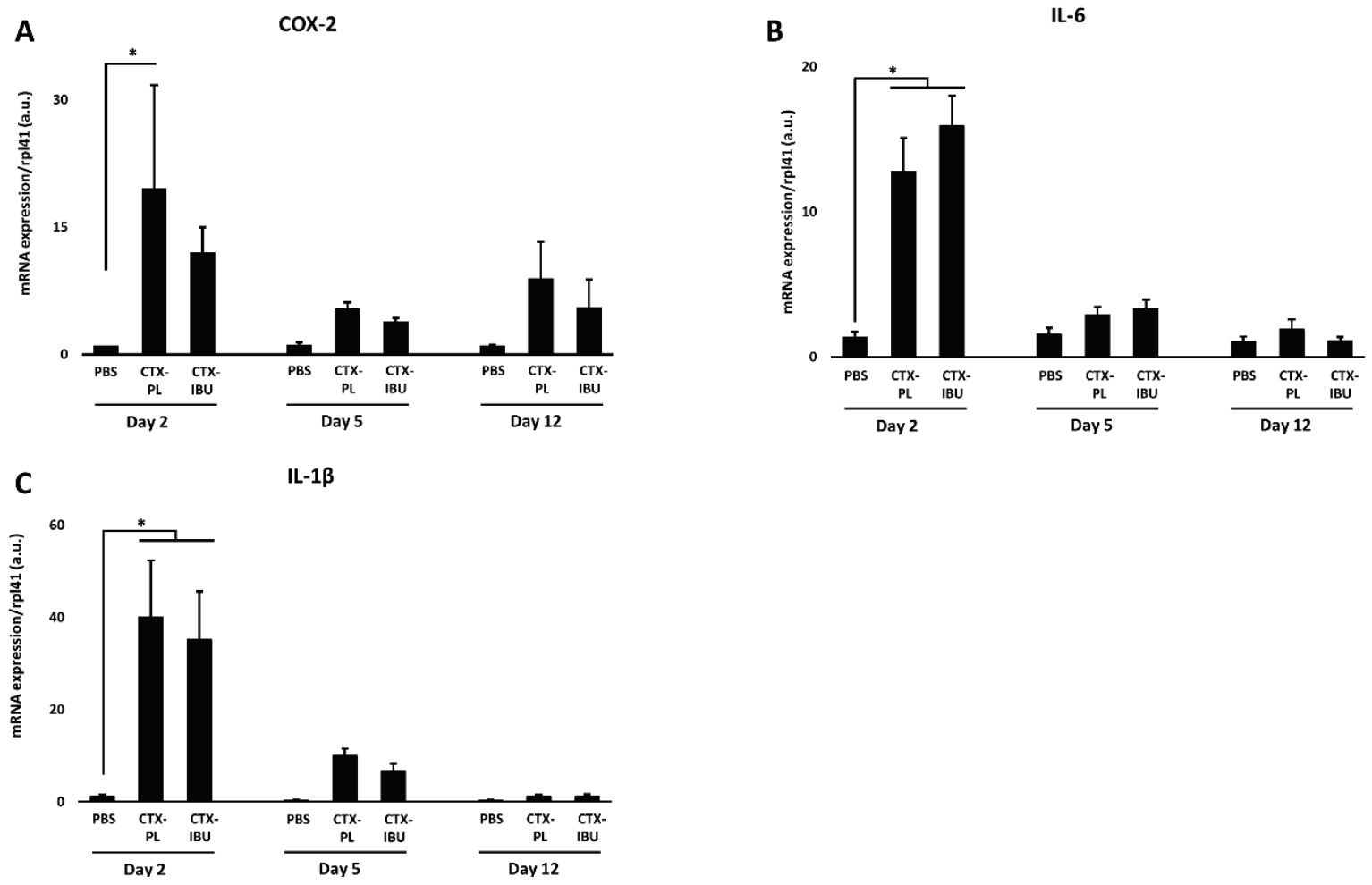

Fig. 5. Data are presented as average \pm SEM for the mRNA expression of inflammatory markers, i.e. cyclooxygenase-2 (COX-2), interleukin-6 (IL-6) and interleukin-1 $\beta$ (IL-1ß). PBS: healthy controls (PBS-injected), CTX-PL: CTX-injected and untreated, CTX-IBU: CTX-injected and ibuprofen-treated. $* p<0.05$.

\section{Muscle anabolism (Fig. 6)}

Members of the mTORC1 signaling pathway were upregulated 2 and 5 days following CTX-injection compared to PBS. At d2, the upstream mediator p-Akt (Fig. 6A) was higher in CTX-IBU (0.47 \pm 0.12 a.u.) and in CTX-PL (0.31 \pm 0.05 a.u.) compared to PBS (0.10 \pm 0.02 a.u.). However, significance was only reached in CTX-IBU ( $p=0.022$ vs. PBS) and not in CTX-PL $(\mathrm{p}=0.353$ vs. PBS). Similarly, compared to PBS (0.29 \pm 0.02 a.u.), p-mTOR (Fig. 6B) expression was higher in CTX-IBU (0.69 \pm 0.09 a.u.; $\mathrm{p}=0.047)$, but not in CTX-PL (0.49 \pm 0.04 a.u.; $p=0.708)$ at $\mathrm{d} 2$. Still, p-mTOR expression did not significantly differ between CTX-PL and CTX-IBU ( $p=0.64)$. Downstream of mTOR, $p-S 6 K 1$ (Fig. 6C) was significantly upregulated 2 days following CTX-injection compared to PBS (0.06 \pm 0.02 a.u.), irrespective of treatment (CTX-PL: $0.58 \pm 0.18$ a.u.; $\mathrm{p}=0.037$; CTX-IBU: $0.70 \pm 0.18$ a.u.; $\mathrm{p}=0.007$ ). Similarly, five days following CTX-injection, $\mathrm{p}$-Akt expression was significantly increased compared to PBS ( $0.29 \pm 0.10$ a.u.), irrespective of treatment (CTX-PL: $1.24 \pm 0.10$ a.u.; p<0.001; CTX-IBU: $1.10 \pm 0.19$ a.u.; p<0.001). A similar pattern was observed for p-mTOR expression, i.e. significantly higher values in CTX-PL (1.15 \pm 0.13 a.u.; $\mathrm{p}<0.001)$ and in CTX-IBU $(1.12 \pm 0.24$ a.u.; $\mathrm{p}<0.001)$ compared to PBS $(0.36 \pm 0.07$ a.u.). Phospho-S6K1 was more elevated in CTX-PL (0.75 \pm 0.21 a.u.; $p=0.008)$ and tended to be higher in CTX-IBU $(0.60 \pm 0.23$ a.u.; $\mathrm{p}=0.066)$ compared to PBS (0.12 \pm 0.05 a.u.). However, there was no difference between CTX-PL and CTX-IBU $(p=1.0)$. Twelve days after the CTX-injection, the phosphorylation state of members of the mTORC1 pathway were similar to PBS. 

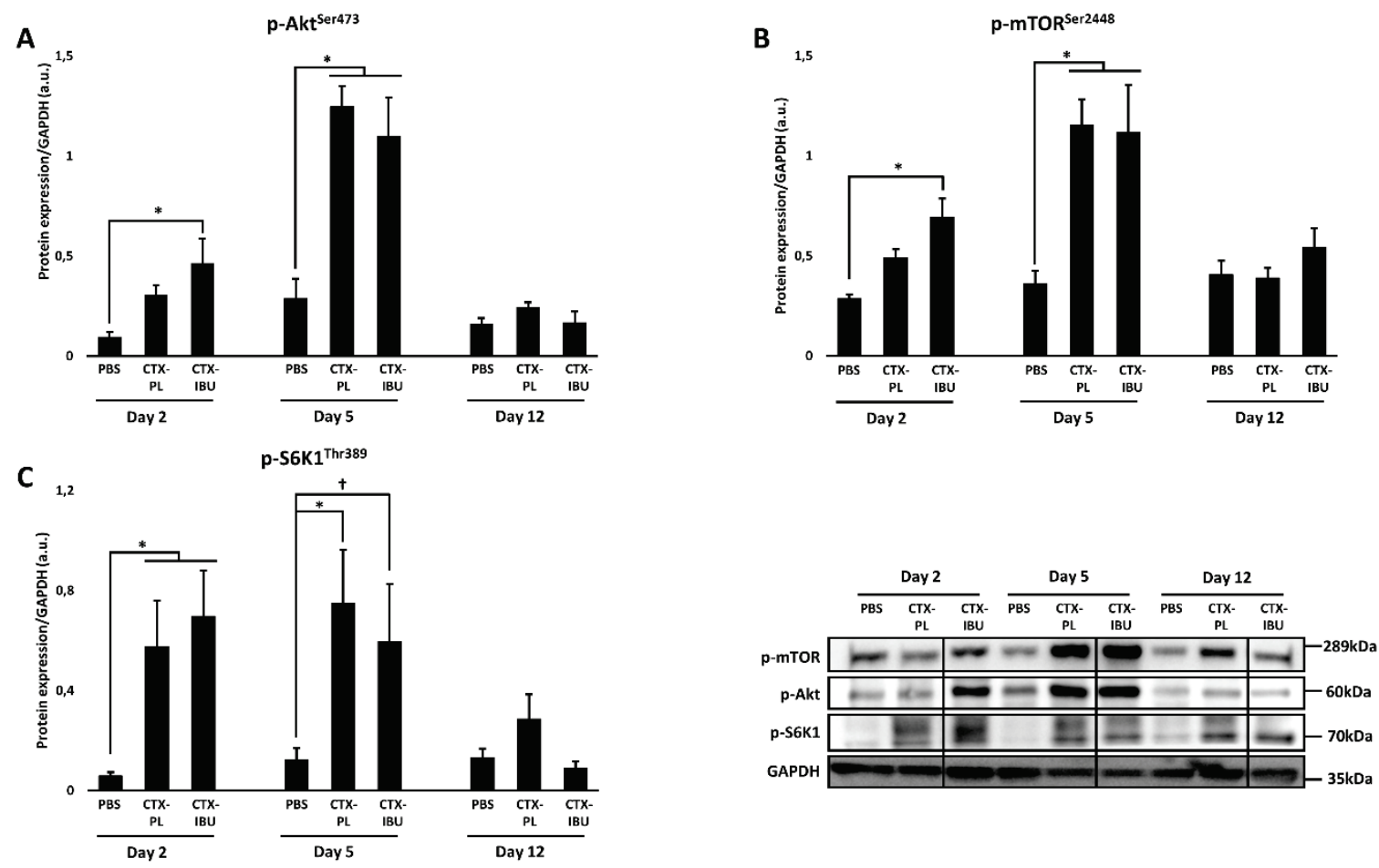

Fig. 6. Data are presented as average \pm SEM for the protein expression of anabolic markers (mTORC1 pathway), i.e. phospho-Akt (p-Akt), p-mammalian target of rapamycin ( $p$-mTOR) and p-ribosomal protein S6 kinase 1 ( $p$-S6K1). PBS: healthy controls (PBS-injected), CTX-PL: CTX-injected and untreated, CTX-IBU: CTX-injected and ibuprofen-treated. ${ }^{*} p<0.05 ;{ }^{\dagger} p=0.05-0.1$.

\section{Discussion}

In the present study, we examined whether a mild dose of ibuprofen impaired muscle regeneration upon injury and whether markers of inflammatory and anabolic signaling were affected. CTX upregulated muscle inflammation in both CTX-PL and CTX-IBU. A mild ibuprofen treatment did not strongly affect the CTX-induced inflammatory signaling. However, whereas Cox-2 expression and $\mathrm{F} 4 / 80^{+}$macrophage infiltration were significantly higher in CTX-PL than in PBS, both parameters were not significantly higher in CTX-IBU vs. PBS. This suggests that mild NSAID treatment upon muscle injury slightly attenuates the CTX-induced muscle inflammatory milieu. Furthermore, ibuprofen also stimulated the mTORC1 pathway. Eventually, none of these ibuprofen-induced changes in inflammatory or anabolic signaling affected muscle regeneration, as the amount and size of regenerating fibers following CTX-injection did not differ between CTX-IBU and CTX-PL. It should be noted that IBU/PL treatment started 3 days before the TA injection. Although it is unlikely that IBU treatment before injury interferes with molecular signaling in the muscle tissue, this does not parallel real life situations in which treatments are implemented after the trauma.
CTX injection severely affected the fiber type composition, i.e. a loss in type IIa muscle fibers at $\mathrm{d} 2$, $\mathrm{d} 5$ and d12. Furthermore, at d2, CTX induced an increase in unstained fibers and a decrease in type IIb muscle fibers. Unstained fibers refer to fibers that express either immature myosin isoforms or the type IIx myosin isoform. Given the increased contribution of unstained fibers very early after injury, it is very likely that this can be attributed to a sudden increase of fibers expressing embryonic and neonatal myosin heavy chains, rather than type IIx fibers (Jerkovic et al. 1997). The loss of type IIb fibers at $\mathrm{d} 2$ and of type IIa fibers at $\mathrm{d} 12$ was partly counteracted by the ibuprofen treatment. This resulted in a muscle phenotype in CTX-IBU that is more similar to the healthy PBS phenotype compared to the untreated injury group. This is the first study that reports a protective effect of ibuprofen on the shift in muscle fiber type following acute injury. One earlier study reported that ibuprofen induced a fiber type shift in rats, i.e. 2 weeks of ibuprofen treatment decreased type IIb and increased IIx muscle fibers during normal cage activity, while type IIa muscle fibers were decreased after a 2-week running protocol ( $5 \times$ per week) (Rooney et al. 2016). This confirms that ibuprofen might indeed interfere with the biomolecular mechanisms which underlie a shift in muscle fiber type. However, future 
studies should further reveal how ibuprofen is involved in the regulation of the fiber type composition and whether this ibuprofen-induced shift is beneficial for muscle recovery and/or functionality.

Muscle traumas, i.e. due to fractures (Hurtgen et al. 2016), strain injuries (Bayer et al. 2018) or excessive exercise (Rubio-Arias et al. 2018), are accompanied by an inflammatory response, which plays a key role in the regeneration process. On the one hand, muscle recovery can be impaired due to diminished inflammation, i.e. through macrophage depletion (Liu et al. 2017, Xiao et al. 2016) or via pharmacological interventions (Bondesen et al. 2004). On the other hand, excessive inflammation, i.e. neutrophil-derived free radicals, might stimulate the skeletal muscle damage (Toumi and Best 2003). Therefore, a tight regulation of inflammatory signaling following injury is of importance to maximize muscle recovery. Lately, much evidence indicates that NSAID use might impair muscle regeneration upon injury (Trappe et al. 2002, Xian and Zhou 2009), but also the increase in muscle mass, muscle strength (Lilja et al. 2017) and protein synthesis following resistance exercise (Trappe et al. 2002). Therefore, it is advised to discontinue prophylactic use of NSAIDs and restrict its use to the minimal dose and duration for the short-term management of acute pain and inflammation (Warden, 2010). The data of the present study suggest that moderate ibuprofen treatment does not impair muscle tissue regeneration. However, it should be noted that the upregulated inflammatory signaling and injury features following CTX-injection (Dalle et al. 2020) are way beyond the inflammatory response upon muscle trauma, e.g. due to sport injury.

It was earlier established that inflammatory signaling negatively impacts muscle metabolism in different contexts such as sepsis (Hasselgren et al. 2005), sarcopenia (Dalle et al. 2017) and injury (Toumi et al. 2006). Therefore, we studied whether suppression of inflammatory signaling affected the markers of muscle anabolic signaling, i.e. the mTORC1 signaling pathway. Our findings suggest that mild ibuprofen-induced selective downregulation of muscle inflammation does not impair mTORC1 signaling upon injury and might even upregulate anabolism during early muscle regeneration. Similarly, in a murine muscle overload model, COX-2 inhibition did not affect mTORC1 signaling (Novak et al. 2009). This might be explained by the 'overloaded' muscle model which is physiologically different from muscle injury. Interestingly, Markworth et al. (2014) observed in healthy young subjects that ibuprofen acutely ingested prior to $(-30 \mathrm{~min} ; 400 \mathrm{mg})$ and following ( +6 and $+12 \mathrm{~h} ; 2 \times 400 \mathrm{mg})$ a resistance exercise bout increased p-S6K $1^{\text {Thr387 }}$ expression $24 \mathrm{~h}$ post-exercise, however, without upregulation of p-Akt ${ }^{\mathrm{Ser} 473}$ (Markworth et al. 2014). In arthritic rats, pharmacological COX-2 inhibition increased the expression of IGF-1 (Granado et al. 2007). Unfortunately, the authors did not look at mTORC1 signaling, which occurs downstream of IGF-1. Also in other cell types, COX-2 signaling was shown to interact with mTORC1 signaling. Treatment of pancreatic $\beta$ cells with $\mathrm{PGE}_{2}$, a downstream effector of COX-2, downregulated p-Akt at Ser473 and Thr308 (Meng et al. 2006). Despite varying contexts, ranging from exerciseinduced muscle damage in humans to CTX-induced muscle injury in mice, these data suggest that there might be a modulatory effect of ibuprofen on muscle anabolic signaling, probably at least partly regulated via COX-2 signaling. Unfortunately, despite its potential role in skeletal muscle protein synthesis (Rodemann and Goldberg 1982), the association between COX-2 and the mTORC1 signaling pathway is barely studied in skeletal muscle tissue. Therefore, it remains to be determined whether modulations of COX signaling in the present study contributed to a stimulation in mTORC1 signaling early upon CTX-injection.

Most evidence indicates that COX-2 inhibition upon muscle injury negatively affects recovery in mice. Similarly to our findings, pharmacological COX-2 inhibition decreased the inflammatory cell infiltration in a freeze-induced muscle injury (Bondesen et al. 2004) and in a muscle overload model (Novak et al. 2009). Contrarily to our findings, this resulted in impaired muscle regeneration, i.e. decreased myofiber size (Bondesen et al. 2004) or muscle mass (Novak et al. 2009). In addition, in vitro evidence indicated that COX-2 inhibition blunted myogenesis in $\mathrm{C}_{2} \mathrm{C}_{12}$ muscle cells. Therefore, it is generally stated that COX-2 inhibition upon muscle injury is very likely to impair muscle recovery. This might be due to PG-dependent inflammatory effects (Prisk and Huard 2003). Furthermore, a downregulation in the PG-induced stimulation of satellite cells (Bondesen et al. 2004, Mendias et al. 2004, Mikkelsen et al. 2009) due to NSAID use might also contribute to the decreased muscle regeneration and muscle strength after muscle repair (Mackey et al. 2012).

In the present study, there was no detrimental 
effect of NSAID use on the skeletal muscle regeneration. The discrepancy between our data and the studies mentioned above might be explained by the use of a different muscle injury/regeneration model. However, the degree of COX-2 inhibition also varied between the studies, due to different doses and the use of different NSAIDs. Despite a dose of $20 \mathrm{mg} \cdot \mathrm{kg}^{-1} \mathrm{BM}$ ibuprofen in our study, Cox-2 mRNA expression was only modestly attenuated ( $40 \%$ ). Substantial lower doses of $6 \mathrm{mg} \cdot \mathrm{kg}^{-1}$ BM SC-236 and $10 \mathrm{mg} \cdot \mathrm{kg}^{-1}$ BM of NS-398 were used in the study of Bondesen et al. (2004) and Novak et al. (2009) respectively. Unfortunately, in these studies, Cox-2 mRNA or protein expression following injury was not compared between an untreated group (e.g. CTX-PL) and a group in which COX-2 was inhibited. Still, it is very likely that their pharmacological COX-2 inhibition was far more potent since the $\mathrm{IC}_{50}$ values for $\mathrm{COX}-2$ activity, though not assessed in skeletal muscle cells, were much lower compared to that of ibuprofen (Bhardwaj et al. 2014, Kato et al. 2001, Kaur et al. 2018). It would be useful if future studies confirm the downregulation of COX-2 following NSAID treatment to ensure that the modifications in muscle regeneration can be mechanistically ascribed to COX-2.

Generally, findings obtained in studies that use very potent COX-2 inhibitors (Bondesen et al. 2004, Markworth and Cameron-Smith 2013, Mendias et al. 2004, Novak et al. 2009) should be interpreted with caution when conclusions are translated to a human setting. Upon injury, people are very likely to use a moderate COX-inhibitor such as ibuprofen, rather than very potent $\mathrm{COX}-2$ inhibitors such as SC-236 and NS-398. Whereas ibuprofen partly inhibits COX-2 and concomitantly attenuates inflammation, it still allows sufficient COX-2 signaling to enable appropriate muscle regeneration. Contrarily, very potent inhibitors block COX-2 signaling to a much higher extent, which does not allow inflammatory processes that are necessary for appropriate myogenesis, e.g. phagocytosis of debris and secretion of growth factors by macrophages, and might therefore impair muscle recovery. Accordingly, when 6-week resistance exercise was combined with chronic naproxen sodium ( $\mathrm{IC}_{50}$ COX-2 (Noreen et al. 1998) and
$\mathrm{PGE}_{2}$ (Gierse et al. 1999) similar to ibuprofen) treatment in healthy males, COX-PG signaling was downregulated (Brewer et al. 2015). Still, this did not affect the muscular adaptations to resistance exercise, confirming that mild, 'pharmacologically-relevant' COX inhibition does not necessarily impair muscular adaptations. Interestingly, in an elderly population, 12 weeks of resistance training combined with chronic ibuprofen supplementation $\left(1.2{\mathrm{~g} . \mathrm{d}^{-1}}^{-1}\right)$ resulted in larger gains in muscle mass and muscle strength compared to an untreated group (Trappe et al. 2011). Future research should further reveal how COX-2 inhibitors with different potencies regulate the inflammation and regeneration upon muscle stress.

NSAID use is very common for the treatment of inflammation and pain, e.g. due to muscle injury. However, different studies raised caution about the use of NSAIDs for muscle recovery purposes, as they impaired regeneration (Bondesen et al. 2004, Mishra et al. 1995, Novak et al. 2009). These studies used strong COX inhibitors to totally abolish muscle inflammatory signaling, and concluded that NSAID use impairs muscle regeneration (Almekinders and Gilbert 1986, Bondesen et al. 2006, Trappe et al. 2002). Although observed in experimental injury models, this might (unjustifiably) discourage muscle injury patients to use this treatment for recovery purposes. In the present study, a moderate COX inhibition through ibuprofen treatment did not oppose muscle regeneration. On the contrary, this treatment seemed to attenuate early inflammatory signaling, i.e. Cox-2 expression and $\mathrm{F} 4 / 80^{+}$macrophage infiltration, and to transiently upregulate markers of muscle anabolism such as p-Akt and p-mTOR. These data suggest that, in contrast to supra-physiologically strong COX inhibitors, there are no reasons to believe that a mild and pharmacologically-relevant ibuprofen treatment impairs the muscle recovery process.

\section{Conflict of Interest}

There is no conflict of interest.

\section{Acknowledgements}

The authors would like to thank Monique Ramaekers for her technical assistance.

\section{References}

ALMEKINDERS LC, GILBERT JA: Healing of experimental muscle strains and the effects of nonsteroidal antiinflammatory medication. Am J Sports Med 14: 303-308, 1986. https://doi.org/10.1177/03635465860140041 
BAYER ML, HOEGBERGET-KALISZ M, JENSEN M, SVENSSON R, COUPE C, BOESEN M, NYBING JD, KURT EY, MAGNUSSON SP, KJAER M: Role of tissue perfusion, muscle strength recovery, and pain in rehabilitation after acute muscle strain injury: A randomized controlled trial comparing early and delayed rehabilitation. Scand J Med Sci Sport 28: 2579-2591, 2018. https://doi.org/10.1111/sms.13269

BHARDWAY A, KAUR J, WUEST F, KNAUS EE: Fluorophore-labeled cyclooxygenase-2 inhibitors for the imaging of cyclooxygenase-2 overexpression in cancer: synthesis and biological studies. ChemMedChem 9: 109-116, 2014. https://doi.org/10.1002/cmdc.201300355

BONDESEN BA, MILLS ST, KEGLEY KM, PAVLATH GK: The COX-2 pathway is essential during early stages of skeletal muscle regeneration. Am J Physiol Cell Physiol 287: C475-C483, 2004. https://doi.org/10.1152/ajpcell.00088.2004

BONDESEN BA, MILLS ST, PAVLATH GK: The COX-2 pathway regulates growth of atrophied muscle via multiple mechanisms. Am J Physiol Cell Physiol 290: C1651-C1659, 2006. https://doi.org/10.1152/ajpcell.00518.2005

BREWER CB, BENTLEY JP, DAY LB, WEDDELL DE, BREWER CB, BENTLEY JP: Resistance exercise and naproxen sodium: effects on a stable PGF2a metabolite and morphological adaptations of the upper body appendicular skeleton. Inflammopharmacology 23: 319-327, 2015. https://doi.org/10.1007/s10787-015-0248-x

CHAZAUD B, SONNET C, LAFUSTE P, BASSEZ G, RIMANIOL AC, PORON F, AUTHIER F-J, DREYFUS PA, GHERARDI RK: Satellite cells attract monocytes and use macrophages as a support to escape apoptosis and enhance muscle growth. J Cell Biol 163: 1133-1143, 2003. https://doi.org/10.1083/jcb.200212046

CHEN S, GERKEN E, ZHANG Y, ZHAN M, MOHAN RK, LI AS, REID MB, LI Y-P: Role of TNFa signaling in regeneration of cardiotoxin-injured muscle. Am J Physiol Cell Physiol 289: C1179-C1187, 2005. https://doi.org/10.1152/ajpcell.00062.2005

CZERWINSKA AM, STREMINSKA W, CIEMERYCH MA, GRABOWSKA I: Mouse gastrocnemius muscle regeneration after mechanical or cardiotoxin injury. Folia Histochem Cytobiol 50: 144-153, 2012. https://doi.org/10.5603/FHC.2012.0021

DALLE S, POFFE C, HIROUX C, RAMAEKERS M, DELDICQUE L, KOPPO K: Cardiotoxin-induced skeletal muscle injury elicits profound changes in anabolic and stress signaling, and muscle fiber type composition. J Muscle Res Cell Motil: 2020 (ahead of print). https://doi.org/10.1007/s10974-020-09584-5

DALLE S, ROSSMEISLOVA L, KOPPO K: The role of inflammation in age-related sarcopenia. Front Physiol 8: 1045, 2017. https://doi.org/10.3389/fphys.2017.01045

ELNACHEF N, SCHEIMAN JM, RICK AMF, HOWDE CWN, CHEY WD: Changing perceptions and practices regarding aspirin, nonsteroidal anti-inflammatory drugs, and cyclooxygenase-2 selective nonsteroidal antiinflammatory drugs among US primary care providers. Aliment Pharmacol Ther 28: 1249-1258, 2008. https://doi.org/10.1111/j.1365-2036.2008.03836.x

FINK E, FORTIN D, SERRURIER B, VENTURA-CLAPIER R, BIGARD AX: Recovery of contractile and metabolic phenotypes in regenerating slow muscle after notexin-induced or crush injury. J Muscle Res Cell Motil 24: 421-429, 2003. https://doi.org/10.1023/A:1027387501614

GARRY G, ANTONY ML, GARRY DJ: Cardiotoxin induced injury and skeletal muscle regeneration. Methods Mol Biol 1460: 191-208, 2016. https://doi.org/10.1007/978-1-4939-3810-0

GIERSE JK, KOBOLDT CM, WALKER MC, SEIBERT K, ISAKSON PC: Kinetic basis for selective inhibition of cyclo-oxygenases. Biochem J 339: 607-614, 1999. https://doi.org/10.1042/bj3390607

GRANADO M, MARTIN AI, VALLANUA A, LOPEZ-CALDERON A: Experimental arthritis inhibits the insulin-like growth factor-I axis and induces muscle wasting through cyclooxygenase-2 activation. Am J Physiol Endocrinol Metab 292: E1656-E1665, 2007. https://doi.org/10.1152/ajpendo.00502.2006.

GREEN GA: Understanding NSAIDs: from aspirin to COX-2. Clin Cornerstone 3: 50-59, 2001. https://doi.org/10.1016/s1098-3597(01)90069-9

HASSELGREN P-O, MENCONI MJ, FAREED MU, YANG H, WEI W, EVENSON A: Novel aspects on the regulation of muscle wasting in sepsis. Int $\mathrm{J}$ Biochem Cell Biol 37: 2156-2168, 2005. https://doi.org/10.1016/j.biocel.2005.01.017 
HURTGEN BJ, WARD CL, GARG K, POLLOT BE, GOLDMAN SM, MCKINLEY TO, WENKE JC, CORONA BT: Severe muscle trauma triggers heightened and prolonged local musculoskeletal inflammation and impairs adjacent tibia fracture healing. J Musculoskelet Neuronal Interact 16: 122-134, 2016.

JARVINEN TA, JARVINEN M, KALIMO H: Early myosin switching induced by nerve activity in regenerating slow skeletal muscle. Cell Struct Funct 22: 147-153, 1997. https://doi.org/10.1247/csf.22.147

KATO M, NISHIDA S, KITASATO H, SAKATA N, KAWAI S: Cyclooxygenase-1 and cyclooxygenase-2 selectivity of non-steroidal anti-inflammatory drugs: investigation using human peripheral monocytes. J Pharm Pharmacol 53: 1679-1685, 2001. https://doi.org/10.1211/0022357011778070

KAUER A, PATHAK SP, SHARMA V, WAKODE S: Synthesis, molecular docking, and pharmacological evaluation derivatives as selective COX-2 inhibitors and anti-inflammatory agents. Arch Pharm Chem Life Sci 351: e1800008, 2018. https://doi.org/10.1002/ardp.201800008

LEFAUCHEUR JP, BILLE ASI: The cellular events of injured muscle regeneration depend on the nature of the injury. Neuromuscul Disord 5: 501-509, 1995. https://doi.org/10.1016/0960-8966(95)00012-c

LENG X, JIANG H: Effects of arachidonic acid and its major prostaglandin derivatives on bovine myoblast proliferation, differentiation, and fusion. Domest Anim Endocrinol 67: 28-36, 2019. https://doi.org/10.1016/j.domaniend.2018.12.006

LILJA M, MANDIC M, APRO W, MELIN M, OLSSON K, ROSENBORG S, GUSTAFSSON T, LUNDBERG TR: High-doses of anti-inflammatory drugs compromise muscle strength and hypertrophic adaptations to resistance training in young adults. Acta Physiol 222: e12948, 2017. https://doi.org/10.1111/ijlh.12426

LIU X, LIU Y, ZHAO L, ZENG Z, XIAO W, CHEN P: Macrophage depletion impairs skeletal muscle regeneration: The roles of regulatory factors for muscle regeneration. Cell Biol Int 41: 228-238, 2017. https://doi.org/10.1002/cbin.10705

MACKEY AL, MIKKELSEN UR, MAGNUSSON SP, KJAER M: Rehabilitation of muscle after injury - the role of antiinflammatory drugs. Scand J Med Sci Sport 22: 8-14, 2012. https://doi.org/10.1111/j.1600-0838.2012.01463.x

MAHDY MAA, LEI HY, WAKAMATSU J-I, HOSAKA YZ, NISHIMURA T: Comparative study of muscle regeneration following cardiotoxin and glycerol injury. Ann Anat 202: 18-27, 2015. https://doi.org/10.1016/j.aanat.2015.07.002

MARKWORTH JF, CAMERON-SMITH D: Prostaglandin F2a stimulates PI3K/ERK/mTOR signaling and skeletal myotube hypertrophy. Am J Physiol Cell Physiol 300: C671-C682, 2011. https://doi.org/10.1152/ajpcell.00549.2009

MARKWORTH JF, CAMERON-SMITH D: Arachidonic acid supplementation enhances in vitro skeletal muscle cell growth via a COX-2-dependent pathway. Am J Physiol Cell Physiol 304: C56-C67, 2013. https://doi.org/10.1152/ajpcell.00038.2012

MARKWORTH JF, VELLA LD, FIGUEIREDO VC, CAMERON-SMITH D, RUSSELL AP: Ibuprofen treatment blunts early translational signaling responses in human skeletal muscle following resistance exercise. J Appl Physiol 117: 20-28, 2014. https://doi.org/10.1152/japplphysiol.01299.2013

MENDIAS CL, TATSUMI R, ALLEN RE: Role of cyclooxygenase-1 and -2 in satellite cell proliferation, differentiation, and fusion. Muscle Nerve 30: 497-500, 2004. https://doi.org/10.1002/mus.20102

MENG ZX, SUN JX, LING JJ, LV JH, ZHU DY: Prostaglandin E2 regulates Foxo activity via the Akt pathway: implications for pancreatic islet beta cell dysfunction. Diabetologia 49: 2959-2968, 2006. https://doi.org/10.1007/s00125-006-0447-5

MIKKELSEN UR, LANGBERG H, HELMARK IC, SKOVGAARD D, ANDERSEN LL, KJAER M, MACKEY AL: Local NSAID infusion inhibits satellite cell proliferation in human skeletal muscle after eccentric exercise. J Appl Physiol 107: 1600-1611, 2009. https://doi.org/10.1152/japplphysiol.00707.2009.

MISHRA DK, FRIDEN J, SCHMITZ MC: Anti-inflammatory medication after muscle injury. A treatment resulting in short-term improvement but subsequent loss of muscle function. J Bone Joint Surg Am 77: 1510-1519, 1995. https://doi.org/10.2106/00004623-199510000-00005

MO C, VALLEJO J, IGWE O, BONEWALD L, WETMORE L, BROTTO M: Prostaglandin E2 promotes proliferation of skeletal muscle myoblasts via EP4 receptor activation. Cell Cycle 14: 1507-1516, 2015. https://doi.org/10.1080/15384101.2015.1026520. 
NOREEN Y, RINGBOM T, PERER P, DANIELSON H, BOHLIN L: Development of a radiochemical cyclooxygenase-1 and -2 in vitro assay for identification of natural products as inhibitors of prostaglandin biosynthesis. J Nat Prod 61: 2-7, 1998. https://doi.org/10.1021/np970343j

NOVAK ML, BILLICH W, SMITH SM, SUKHIJA KB, MCLOUGHIN TH, HORNBERGER TA, KOH TJ: COX-2 inhibitor reduces skeletal muscle hypertrophy in mice. Am J Physiol Regul Integr Comp Physiol 296: R1132-R1139, 2009. https://doi.org/10.1152/ajpregu.90874.2008

PRISK V, HUARD J: Muscle injuries and repair: The role of prostaglandins and inflammation. Histol Histopathol 18: 1243-1256, 2003. https://doi.org/10.14670/HH-18.1243

RODEMANN HP, GOLDBERG AL: Arachidonic acid, prostaglandin E2, and F2 $\alpha$ influence rates of protein turnover in skeletal and cardiac muscle. J Biol Chem 257: 1632-1638, 1982.

RODEMANN HP, WAXMAN L, GOLDBERG AL: The stimulation of protein degradation in muscle by Ca2+ is mediated by prostaglandin E2 and does not require the calcium-activated protease. J Biol Chem 257: 8716-8723, 1982.

ROONEY SI, BASKIN R, TORINO DJ, VAFA RP, KHANDEKAR PS, KUNTZ AF, SOSLOWSKY LJ: Ibuprofen differentially affects supraspinatus muscle and tendon adaptations to exercise in a rat model. Am J Sport Med 44: 2237-2245, 2016. https://doi.org/10.1177/0363546516646377

RUBIO-ARIAS JA, AVILLA-GANDIA V, LOPEZ J, SOTO-MENDEZ D, ALCARAZ PE, RAMOS-CAMPO DJ: Muscle damage and inflammation biomarkers after two ultra-endurance mountain races of different distances: 54 km vs 111 km. Physiol Behav 205: 51-57, 2018. https://doi.org/10.1016/j.physbeh.2018.10.002

TIDBALL JG: Inflammatory processes in muscle injury and repair. Am J Physiol Integr Comp Physiol 288: R345-R353, 2005. https://doi.org/10.1152/ajpregu.00454.2004

TOUMI H, BEST TM: The inflammatory response: friend or enemy for muscle injury? Br J Sport Med 37: 284-286, 2003. https://doi.org/10.1136/bjsm.37.4.284

TOUMI H, F'GUYER S, BEST TM: The role of neutrophils in injury and repair following muscle stretch. J Anat 208: 459-470, 2006. https://doi.org/10.1111/j.1469-7580.2006.00543.x

TRAPPE TA, CARROLL CC, DICKINSON JM, LEMOINE JK, HAUS JM, SULLIVAN BE: Influence of acetaminophen and ibuprofen on skeletal muscle adaptations to resistance exercise in older adults. Am J Physiol Regul Integr Comp Physiol 300: R655-R662, 2011. https://doi.org/10.1152/japplphysiol.01348.2010

TRAPPE TA, WHITE F, LAMBERT CP, CESAR D, HELLERSTEIN M, EVANS WJ: Effect of ibuprofen and acetaminophen on postexercise muscle protein synthesis. Am J Physiol Endocrinol Metab 282: E551-E556, 2002. https://doi.org/10.1152/ajpendo.00352.2001

VANDENBURGH H, HATFALUDY S, SOHAR I, SHANSKY J: Stretch-induced prostaglandins and protein turnover in cultured skeletal muscle. Am J Physiol Cell Physiol 259: C232-C240, 1990. https://doi.org/10.1152/ajpcell.1990.259.2.c232

WARDEN SJ: Prophylactic use of NSAIDs by athletes: a risk/benefit assessment. Phys Sportsmed 38: 132-138, 2010. https://doi.org/10.3810/psm.2010.04.1770

WEINHEIMER EM, JEMIOLO B, CARROLL CC, HARBER MP, HAUS JM, BURD NA, LEMOINE JK, TRAPPE SW, TRAPPE TA: Resistance exercise and cyclooxygenase (COX) expression in human skeletal muscle: implications for COX-inhibiting drugs and protein synthesis. Am J Physiol Regul Integr Comp Physiol 292: R2241-R2248, 2007. https://doi.org/10.1152/ajpregu.00718.2006

XIAN CJ, ZHOU X: Treating skeletal pain: limitations of conventional anti-inflammatory drugs, and anti-neurotrophic factor as a possible alternative. Nat Rev Rheumatol 5: 92-98, 2009. https://doi.org/10.1038/ncprheum0982

XIAO W, LIU Y, CHEN P: Macrophage depletion impairs skeletal muscle regeneration: the roles of pro-fibrotic factors, inflammation, and oxidative stress. Inflammation 39: 2016-2028, 2016. https://doi.org/10.1007/s10753-016-0438-8 